OPEN ACCESS

Edited by:

Roy D. Sleator,

Cork Institute of Technology, Ireland

Reviewed by:

Huiluo Cao,

The University of Hong Kong,

Hong Kong

Pat G. Casey,

University College Cork, Ireland

${ }^{*}$ Correspondence:

Xiang Xiao

xoxiang@sjtu.edu.cn

Specialty section

This article was submitted to

Evolutionary and Genomic

Microbiology,

a section of the journal

Frontiers in Microbiology

Received: 12 May 2015

Accepted: 26 October 2015

Published: 10 November 2015

Citation:

He Y, Feng $X$, Fang J, Zhang $Y$ and Xiao $X$ (2015) Metagenome and Metatranscriptome Revealed a Highly Active and Intensive Sulfur

Cycle in an Oil-Immersed

Hydrothermal Chimney in Guaymas Basin. Front. Microbiol. 6:1236. doi: 10.3389/fmicb.2015.01236

\section{Metagenome and Metatranscriptome Revealed a Highly Active and Intensive Sulfur Cycle in an Oil-Immersed Hydrothermal Chimney in Guaymas Basin}

\author{
Ying He ${ }^{1,2}$, Xiaoyuan Feng ${ }^{1}$, Jing Fang ${ }^{1}$, Yu Zhang ${ }^{1,2,3}$ and Xiang Xiao ${ }^{1,2,3 *}$ \\ ${ }^{1}$ State Key Laboratory of Microbial Metabolism, School of Life Sciences and Biotechnology, Shanghai Jiao Tong University, \\ Shanghai, China, ${ }^{2}$ State Key Laboratory of Ocean Engineering, Shanghai Jiao Tong University, Shanghai, China, ${ }^{3}$ Institute of \\ Oceanology, Shanghai Jiao Tong University, Shanghai, China
}

The hydrothermal vent system is a typical chemosynthetic ecosystem in which microorganisms play essential roles in the geobiochemical cycling. Although it has been well-recognized that the inorganic sulfur compounds are abundant and actively converted through chemosynthetic pathways, the sulfur budget in a hydrothermal vent is poorly characterized due to the complexity of microbial sulfur cycling resulting from the numerous parties involved in the processes. In this study, we performed an integrated metagenomic and metatranscriptomic analysis on a chimney sample from Guaymas Basin to achieve a comprehensive study of each sulfur metabolic pathway and its hosting microorganisms and constructed the microbial sulfur cycle that occurs in the site. Our results clearly illustrated the stratified sulfur oxidation and sulfate reduction at the chimney wall. Besides, sulfur metabolizing is closely interacting with carbon cycles, especially the hydrocarbon degradation process in Guaymas Basin. This work supports that the internal sulfur cycling is intensive and the net sulfur budget is low in the hydrothermal ecosystem.

Keywords: hydrothermal vent, metagenomics, metatranscriptomics, sulfur cycle, carbon cycle

\section{INTRODUCTION}

Hydrothermal vents are often discovered in ocean ridges where hydrothermal fluid is emitted after the hydrothermal circulation and alteration of seawater entrained through geothermally heated subseafloor basalt (Von Damm, 1990). The deep-sea hydrothermal vent fluid is commonly characterized by its high temperature, varied salinity, enriched metallic elements, and particularly high contents of reduced chemicals, such as $\mathrm{H}_{2}, \mathrm{CH}_{4}$, and $\mathrm{H}_{2} \mathrm{~S}$ (Jannasch and Mottl, 1985). A thermodynamic non-equilibrium is created when the hydrothermal vent fluid encounters sea water that is cold and at a rather high oxidative state, which allows various abiotic and biotic reactions occur. Thus, the hydrothermal vent system is a typical chemosynthetic ecosystem in which microorganisms play essential roles in the generation, consumption, and modification of energy available in the environment (Reysenbach and Shock, 2002). 
In the hydrothermal vent ecosystem, almost all types of inorganic sulfur compounds (e.g., $\mathrm{S}^{2-}, \mathrm{S}, \mathrm{S}_{2} \mathrm{O}_{2}{ }^{2-}, \mathrm{SO}_{2}, \mathrm{~S}_{2} \mathrm{O}_{3}{ }^{2-}$, and $\mathrm{SO}_{4}{ }^{2-}$ ) are abundant and actively converted through chemosynthetic pathways to provide energy and thus sustain the microbial population in the ecosystem (Nakagawa et al., 2005). For example, in the Lost City hydrothermal field, the dominant Thiomicrospira-like group, which consists of sulfuroxidizing chemolithoautotrophs, was observed in the carbonate chimney (Brazelton and Baross, 2010). In the Lau Basin hydrothermal vent field, sulfur-oxidizing Alphaproteobacteria, Gammaproteobacteria, and Epsilonproteobacteria have been suggested to be dominant in the exterior chimney, whereas putative sulfur-reducing Deltaproteobacteria are dominant in the interior of the chimney (Sylvan et al., 2013). In the Guaymas Basin hydrothermal vent field, sulfate-reducing microorganisms, e.g., Desulfobacterales, have been detected and are hypothesized to be involved in the anaerobic methane-oxidation process (Biddle et al., 2012). Moreover, the sulfur cycling is alternated by the chemical reactions that occur during the emitting and growth of the hydrothermal vent. Reduced sulfur compounds are extremely sensitive to oxidants and easily precipitated with metal ions to form chimney or nodule structures (Orcutt et al., 2011). Moreover, shifts in temperature and fluid composition have been observed during the life span of a hydrothermal vent. For example, at $9^{\circ} \mathrm{N}$ East Pacific Rise, Bio9 vent fluids were $368^{\circ} \mathrm{C}$ in 1991 , increased to an estimated temperature greater than or equal to $388^{\circ} \mathrm{C}$ after a second volcanic event in 1992, and thereafter declined over the next similar to 2 years reaching a temperature of $365^{\circ} \mathrm{C}$ in December 1993 (Fornari et al., 1998). The hydrogen concentration in the hydrothermal plum in the NE Lau Basin dropped from $14843 \mathrm{nM}$ in 2008 to $4410 \mathrm{nM}$ in 2010 then further to $7 \mathrm{nM}$ in 2012 (Baumberger et al., 2014). As a result, environmental fluctuations may be induced between sulfateand sulfur-reducing archaea and contribute to the diverse roles of these microorganisms in the ecosystem (Teske et al., 2014). Therefore, a better understanding of sulfur cycling is essential for describing the geobiochemistry and providing hints to identify the life status of a hydrothermal vent ecosystem.

Due to the complexity of microbial sulfur cycling resulting from the numerous parties involved in the process, the sulfur budget in a hydrothermal vent is poorly characterized. To date, most studies have focused on the abundance and diversity of sulfur oxidizers and sulfate reducers in environmental samples through a metagenomic approach (Nakagawa et al., 2005). The exception is the study conducted by Anantharaman et al. (2013), who combined metatranscriptomic and metagenomic analyses of a hydrothermal plume sample and demonstrated the novel metabolic potentials of the SUP05 group of uncultured sulfur-oxidizing Gammaproteobacteria. However, this finding is based on the near-complete genomes of two SUP05 populations, and the information is restricted to this particular group of sulfur oxidizers (Anantharaman et al., 2013). The in-depth mining of the metatranscriptomic data remains too scarce to allow construction of the entire sulfur cycle and thus further illustrate the interactions of this process with the biological cycling of $\mathrm{C}, \mathrm{N}$, and $\mathrm{O}$ elements.

The Guaymas Basin in the Gulf of California is a young marginal rift basin characterized by the active hot venting of reduced sulfur compounds and the rapid deposition of organic-rich sediments. These features make the sulfur cycle in this ecosystem particularly intensive and closely interact with the carbon cycle, including hydrocarbon degradation (Bergmann et al., 2011). Thus, this sampling site is ideal for illustrating all of the possible microbial sulfur metabolic pathways and to evaluate the maximal biomass contribution of sulfur-metabolizing microorganism to the hydrothermal vent ecosystem. In this study, we performed an integrated metagenomic and metatranscriptomic analysis on a chimney sample from Guaymas Basin to achieve a comprehensive study of each sulfur metabolic pathway and its hosting microorganisms and constructed the microbial sulfur cycle that occurs in the site.

\section{RESULTS}

\section{Composition of the Microbial Community}

The composition and function of this microbial community were assessed at both the DNA and RNA levels to estimate the community metabolic potential and activity, respectively. The metagenome and metatranscriptome sequencing resulted in $199,903,215$ and 1,885,022,958 bp clean sequences, respectively (Table 1). The metagenome raw reads were assembled into 49,055 contigs with an average length of $544 \mathrm{bp}$. In total, 5,417,253 reads (26.2\%) from the metatranscriptome were mapped onto metagenomic contigs for quantification of the gene transcripts. 222 and 690,059 16S rRNA gene fragments were identified from the metagenome and metatranscriptome, respectively. The class-level taxonomic compositions of the metagenome and metatranscriptome revealed obvious differences in the presence and the activity of microbes in this community (Table 1). At the DNA level (Figure 1A), Archaeoglobi were found to be the most abundant, with $24.0 \%$ of the sequences assigned, and followed by Deltaproteobacteria (23.6\%) and

TABLE 1 | Summary of the metagenome and metatranscriptome.

\begin{tabular}{lcc}
\hline & Metagenome & Metatranscriptome \\
\hline Size of raw reads (bp) & $199,903,215$ & $1,885,022,958$ \\
Total no. of raw reads & 512,830 & $20,714,538$ \\
Size of assembled contigs (bp) & $26,703,275$ & - \\
Total assembled contigs & 49,055 & - \\
Average contig length (bp) & 544 & - \\
Average GC content of assembled & 43 & - \\
contigs (\%) & & \\
Total no. of genes encoding in the & 53,034 & $5,417,253$ \\
contigs & & \\
Total no. of metatranscriptomic reads & - & 690,059 \\
mapped to the metagenome & & \\
Total no. of $16 S$ rRNA sequences & 222 &
\end{tabular}


A

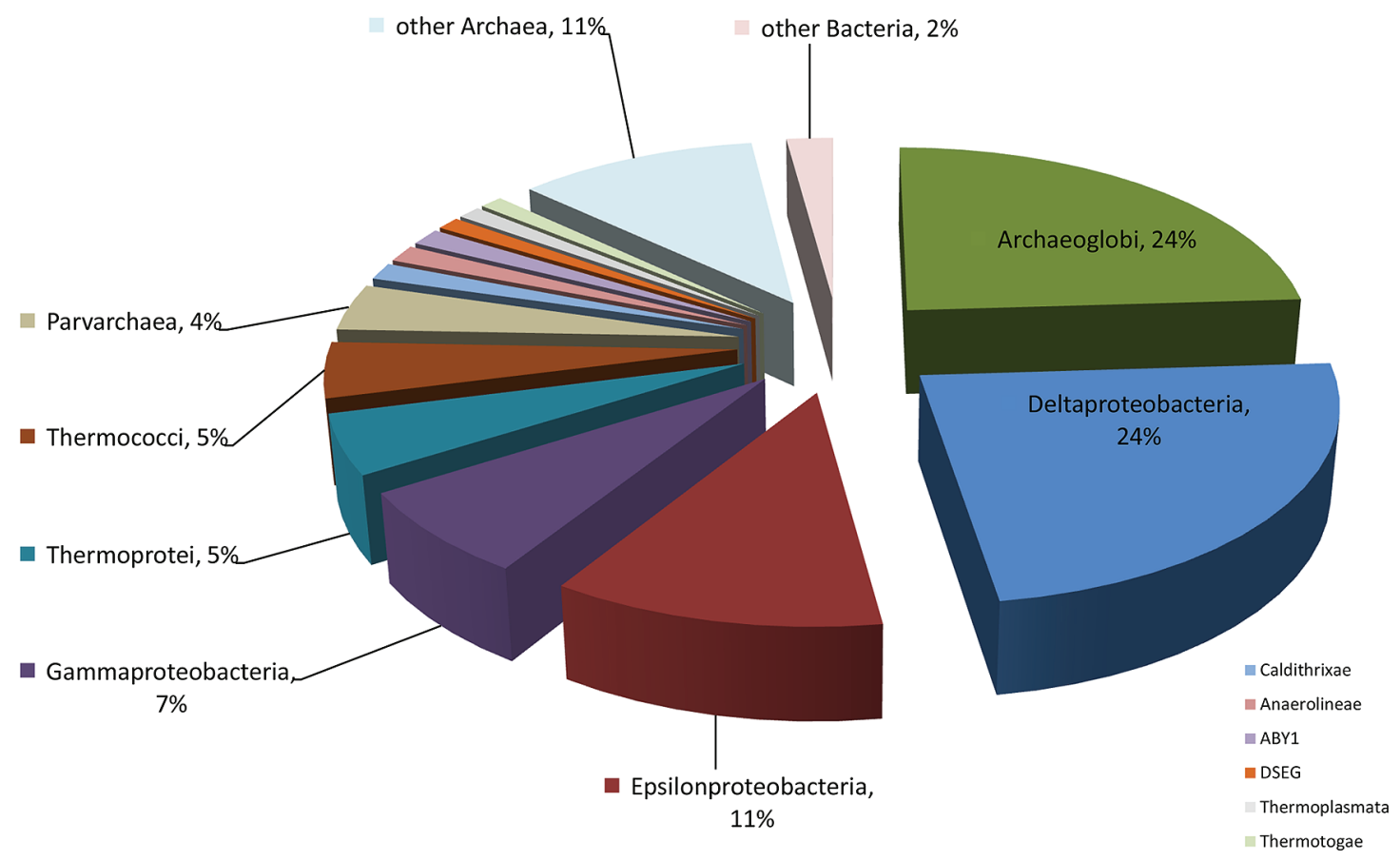

B

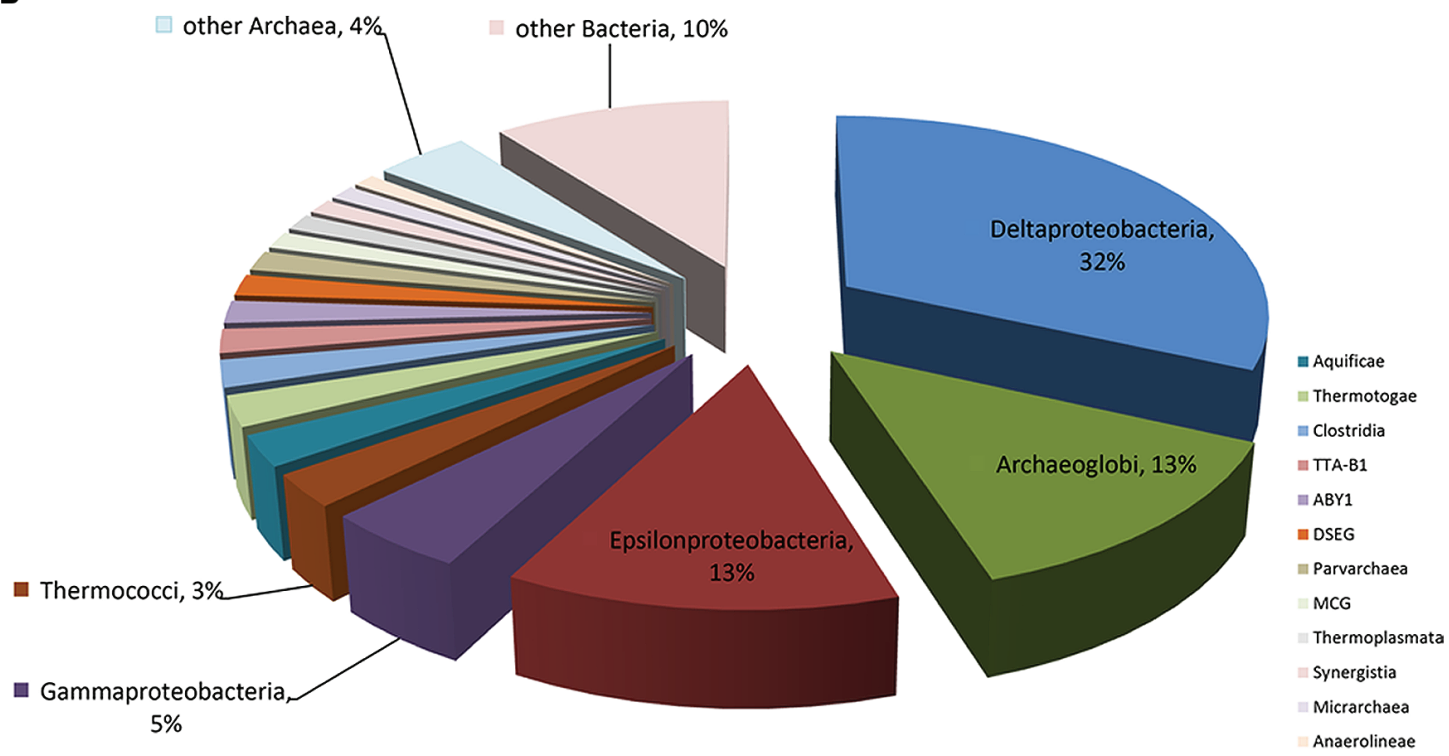

FIGURE 1 | Microbial composition of the enriched AOM-SR community. Detailed information is displayed in Table 1. (A) Percentage of the microbial community determined from the 16S rRNA gene sequences retrieved from the metagenome. (B) Percentage of the microbial community determined from the 16S rRNA gene sequences retrieved from the metatranscriptome.

Epsilonproteobacteria (11.3\%). At the RNA level (Figure 1B), the same dominant groups were found: Deltaproteobacteria (31.8\%), Archaeoglobi (13.3\%), and Epsilonproteobacteria (12.8\%). As reported previously ( $\mathrm{He}$ et al., 2013), 53,034 gene features were predicted and then followed by manual examination and 19,491 gene features (36.8\%) were considered to have expressions determined by transcriptomic reads mapping (see Materials and Methods). A total of 8929 (45.3\%) and 4628 (23.7\%) of all of the expressed genes were assigned (based on the BLAST results as described in Section "Materials and Methods") to Bacteria and Archaea, respectively, and the remaining sequences were not assigned to any category. Among 
the 13,557 expressed genes with taxonomic information, 2135 (15.7\%) were from the highly abundant Archaeoglobi, which is consistent with the results from the 16S rRNA gene analysis. Although the assignment of bacterial genes could not be resolved well at the family level, the dominance of Deltaproteobacteria and Epsilonproteobacteria was still observed. As the archaeal cells typically have fewer copies of the 16S rRNA gene compared with bacterial cells, the proportion of active Archaeoglobi in this community was underestimated. Nevertheless, the predominant active players in this microbial community were Deltaproteobacteria, Archaeoglobi, and Epsilonproteobacteria.

The de novo assembly of metagenomic reads and binning by tetranucleotide signatures (Dick et al., 2009) identified three genomic bins (Supplementary Figure S1 and Supplementary Table S1). These three bins (herewith denoted bin20, bin21, and bin22) were assigned based on their phylogenomic marker genes to Desulfobacteraceae, Desulfovibrionales and Archaeoglobus. The identified genes in the obtained bins ranged from 486 to 1224 . The genome completeness was estimated to range from $\sim 10$ to $34 \%$, based on singlecopy gene estimation (Supplementary Table S1). These three genomic bins will improve the taxonomic assignment of the expressed genes and the reconstruction of the metabolic pathways.

\section{Sulfur Metabolism}

The genes involved in the oxidation of reduced sulfur (ORS) are sulfide quinone oxidoreductase $(s q r)$, which mediates the oxidation of sulfide $\left(\mathrm{HS}^{-}\right)$to elemental sulfur $\left(\mathrm{S}^{0}\right)$, the Sox enzyme complex (soxABXYZ), which is responsible for the oxidation of thiosulfate $\left(\mathrm{S}_{2} \mathrm{O}_{3}{ }^{2-}\right)$ to elemental sulfur, the reverse dissimilatory sulfite reductase complex $(r d s r)$, which is responsible for the oxidation of elemental sulfur to sulfite $\left(\mathrm{SO}_{3}{ }^{2-}\right)$, and adenosine $5^{\prime}$-phosphosulfate reductase $(a p r)$ and sulfate adenylyltransferase (sat) for oxidation of sulfite to sulfate $\left(\mathrm{SO}_{4}{ }^{2-}\right.$; Anantharaman et al., 2013). Conversely, the genes associated with the dissimilatory sulfate reduction (DSR) pathway (Fritz et al., 2002) are sat, apr, and sulfite reductase $(d s r)$. The repertoire of genes associated with the ORS and DSR pathways were found to be expressed in this community (Table 2). Both $a p r$ and $d s r$ were found at high expression levels in bin21 and bin22, confirming their active presence in SRB and Archaeoglobus. The sqr gene, key gene in the ORS pathway, is found present and active in Epsilonproteobacteria, of which the most highly expressed representative was classified into Sulfurimonas (Figure 2) that is one of the most abundant sulfur-oxidizing bacteria found in hydrothermal vent chimneys (Cao et al., 2014). The sox genes were not identified in either the metagenome or metatranscriptome (Table 2). In Epsilonproteobacteria, the proposed microorganism in the present study to perform the ORS pathway, sat gene was found to exhibit high and medium expression levels (Table 2). However, either $a \operatorname{pr} A B$ or $d s r A B$ was identified in the metagenome or metatranscriptome. This finding may be due to the fact that the 454-based metagenomes are still with low coverage and unable to present all the important functional genes. In

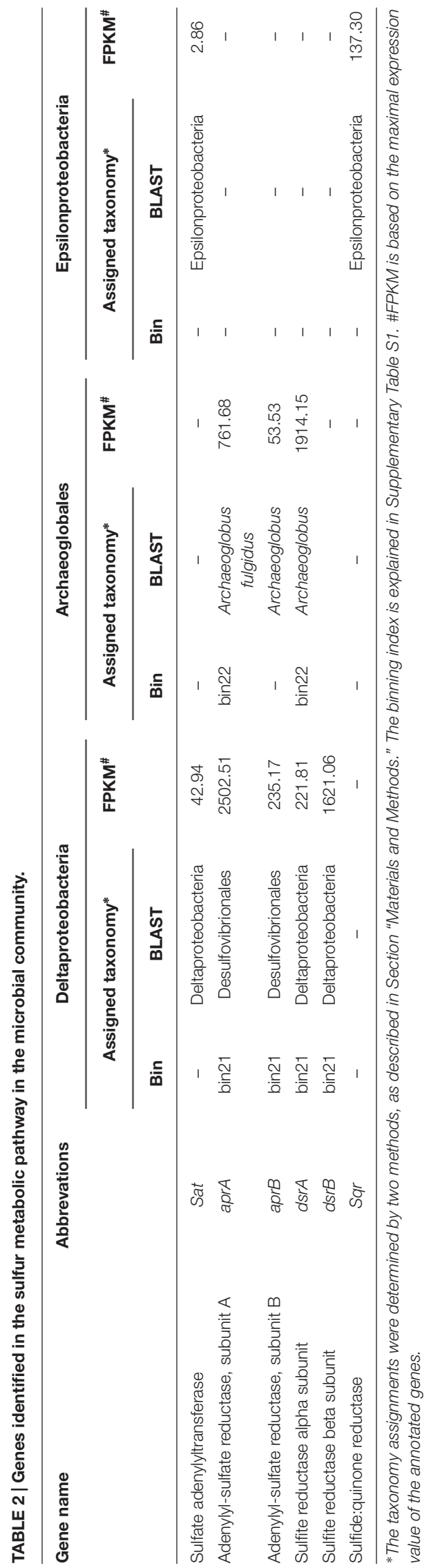




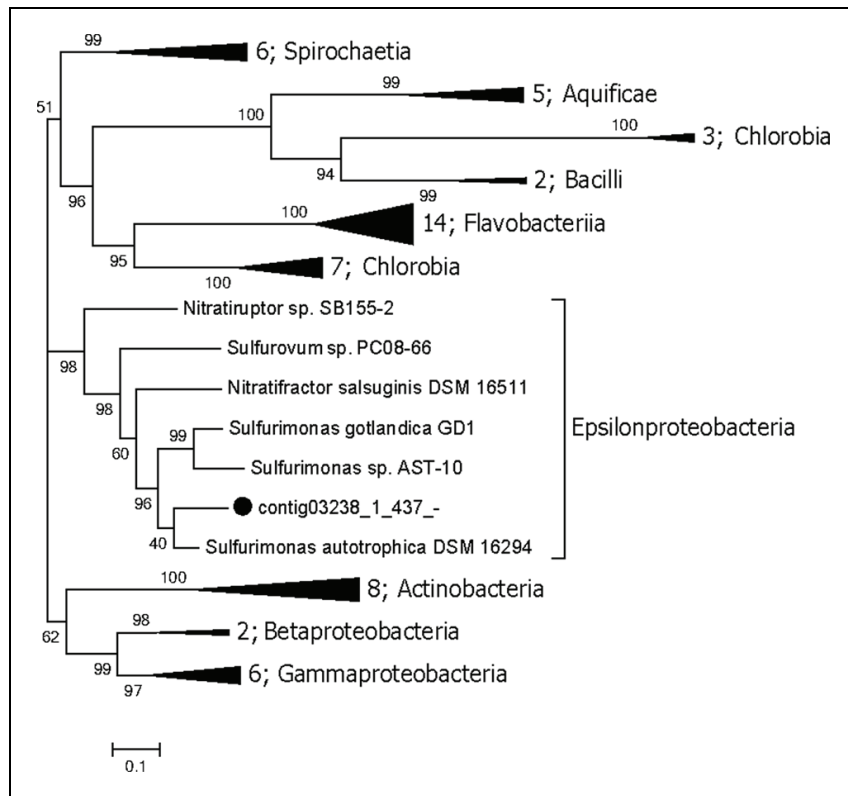

FIGURE 2 | Maximum likelihood phylogenetic tree of the sqr genes.

The bootstrap values are based on 1000 replicates, and the percentages are shown at the nodes. The genes identified in this study are highlighted with black dots. Numbers of genomes in each collapsed clade are displayed before the clade name.

Deltaproteobacteria and Archaeoglobales, which were proposed to conduct the DSR pathway in this study, $\operatorname{apr} A B$ and $d s r A$ genes were found to be highly expressed in both of these two taxonomic groups, whereas sat and $d s r B$ genes were found only in Deltaproteobacteria. The phylogenies of aprA and $d s r A$ further confirmed their assignment to Deltaproteobacteria (Supplementary Figures S2A,B). In a previous study, the aprA with the highest abundance was assigned to the genus Desulfobulbus (Cao et al., 2014). In our study, the aprA gene with the highest expression was assigned to Desulfovibrio (Supplementary Figure S2A). To summarize, the taxonomic assignment and expression of key genes in the sulfur cycle suggest that both the ORS and DSR pathways are highly active in this oil-immersed microbial community, and the energy generated by the sulfur metabolism supports the dominant and active group (Figure 3).

Because there are no metatranscriptome published for any hydrothermal vent chimneys, we compared the expression patterns of the sulfur-metabolizing genes in this metatranscriptome to those in the available metatranscriptome of a plum sample that was also collected from Guaymas Basin (Lesniewski et al., 2012). As shown in Figure 4, sulfur metabolizing (including oxidation and reduction) genes were among the most abundant genes found in the metatranscriptome, and a significant difference ( $p$ value $<0.001)$ in the expression profiles of sulfur metabolizing genes was observed between the chimney and the plume metatranscriptome. Therefore, the sulfur-metabolizing genes were highly abundant and expressed in this GB chimney sample, and displayed significantly higher expression pattern than those of a hydrothermal vent plume sample from Guaymas Basin.

\section{Carbon Metabolism}

In this study, the complete WL pathway was identified in Archaeoglobales with high expression levels (Supplementary Table S2). The CBB cycle was not identified. The genes involved in the complete rTCA cycle were found to be actively present in both Deltaproteobacteria and Epsilonproteobacteria that dominated this chimney microbial community (Table 3). The key gene in the rTCA cycle, ATP-citrate lyase ( $a c l)$, identified in this study to exhibit the highest expression was from Epsilonproteobacteria and exhibited the highest similarity to Sulfurovum, a novel sulfur-, nitrate-, and thiosulfate-reducing and strictly anaerobic chemolithoautotroph bacterium isolated from a deep-sea hydrothermal vent chimney at the Central Indian Ridge (Mino et al., 2014). In this study, the key enzyme for the utilization of acetate, acetyl-CoA synthetase (acd/acs), was found to be expressed and was assigned to sulfate-reducing bacteria (SRB; bin21 as shown in Table 3). In addition, the rTCA cycle and WL pathway were found to be the main pathways for carbon fixation by the dominant Bacteria and Archaea, respectively. This result suggests that, in combination with sulfur metabolism, autotrophic carbon fixation may play an important role in the survival and dominance of these species in the community. Moreover, as shown in Supplementary Table S3, genes involved in the flagellar assembly process were found to be actively present in Desulfovibrionales (bin21). The active role of the flagellar system in SRB may facilitate the movement toward electron donors and nutrients that occurs under the highly fluctuating conditions resulting from eruptions of hydrothermal vents. $\mathrm{SRB}$ have been reported to have the potential to anaerobically oxidize diverse hydrocarbons, such as alkanes, in Guaymas Basin sediments and chimney samples (Rueter et al., 1994). In this study, the activity and expression level of the presumably key gene in fumarate addition, a process through which alkanes are added to the double bond of fumarate based on the activity of alkylsuccinate synthase (ass), was checked. The ass genes were found to be highly active in this community, as determined through their expression level, and the most highly expressed hits were from Desulfoglaeba alkanexedens (Agrawal and Gieg, 2013), a typical sulfate-reducing and alkane-oxidizing bacterium (Supplementary Table S4). Moreover, the enzymes required for the degradation of a variety of organic compounds, such as hydrocarbons, fatty acids, chitins and proteins, have been detected in both the metagenome and metatranscriptome (Supplementary Table S5). Despite their important roles in carbon and global sulfur cycle, the energy metabolism of $\mathrm{SRB}$ remains poorly understood. After taxonomic assignment (see Materials and Methods), cyctochrome c (cytC), formate dehydrogenase $(f d h)$, F-type ATPase (atp), NADH-quinone oxidoreductase (nuo), electron transport complex protein ( $r n f)$ and hydrogenases, such as $\mathrm{Ni} / \mathrm{Fe}$-hydrogenase I (hyaAB) and hydrogenase nickel incorporation and accessory protein (hypA and $h y p($ ), were found with expressions and assigned to SRB (Supplementary Table S6). The presence of hydrogenases and $f d h$ 


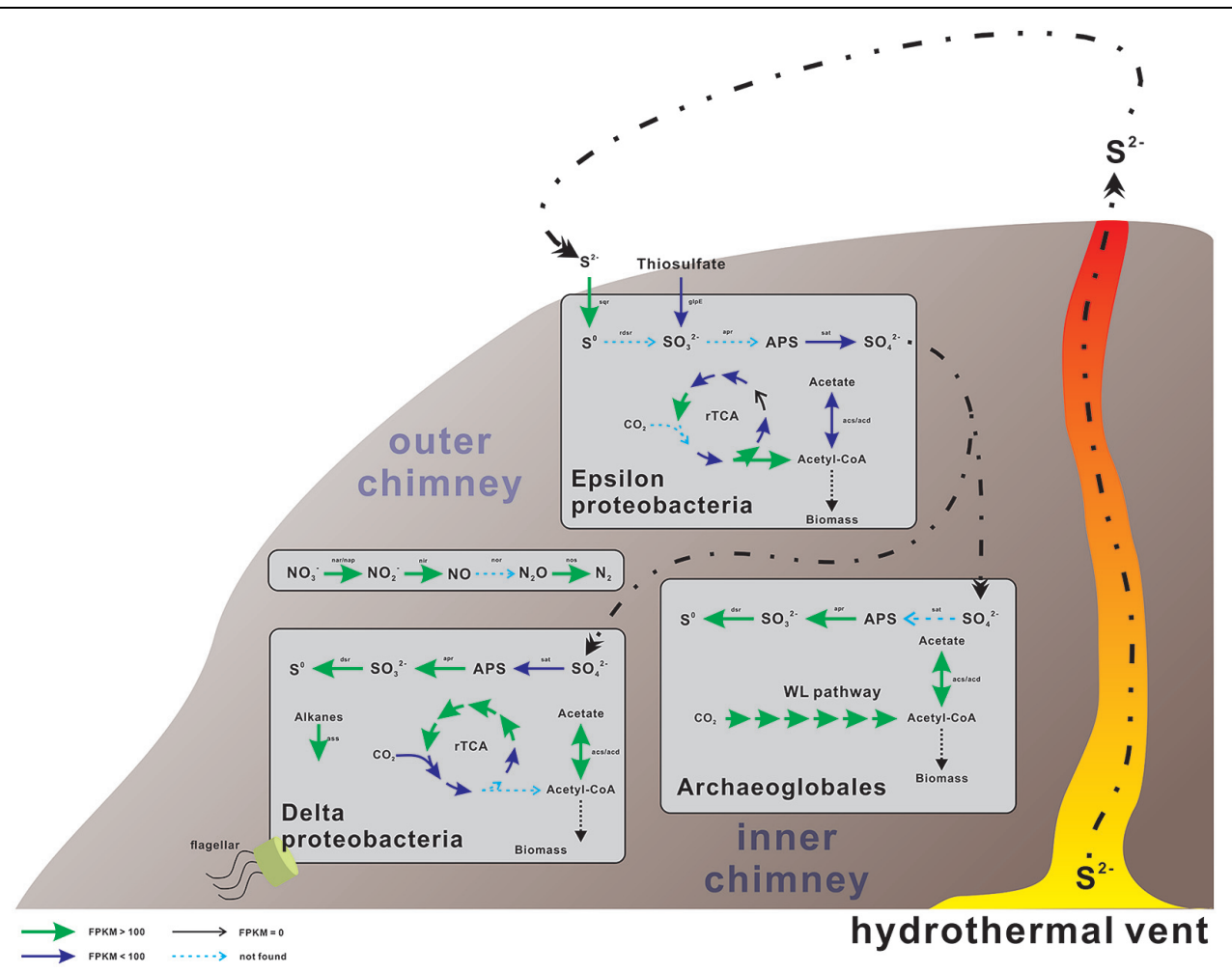

FIGURE 3 | Proposed metabolic and active pathways in this chimney community. The expression and presence of the genes are indicated by the color and thickness of the arrows, as shown in the bottom-right side bar. For genes with multiple hits, only the genes with the highest expression value (FPKM, expected fragments per kilobase of transcript per million fragments mapped) are displayed and discussed. The processes conducted by Archaea are shown on the left, whereas those conducted by bacteria are presented on the right. Detailed information of these genes is displayed in Tables $\mathbf{2 - 4}$.

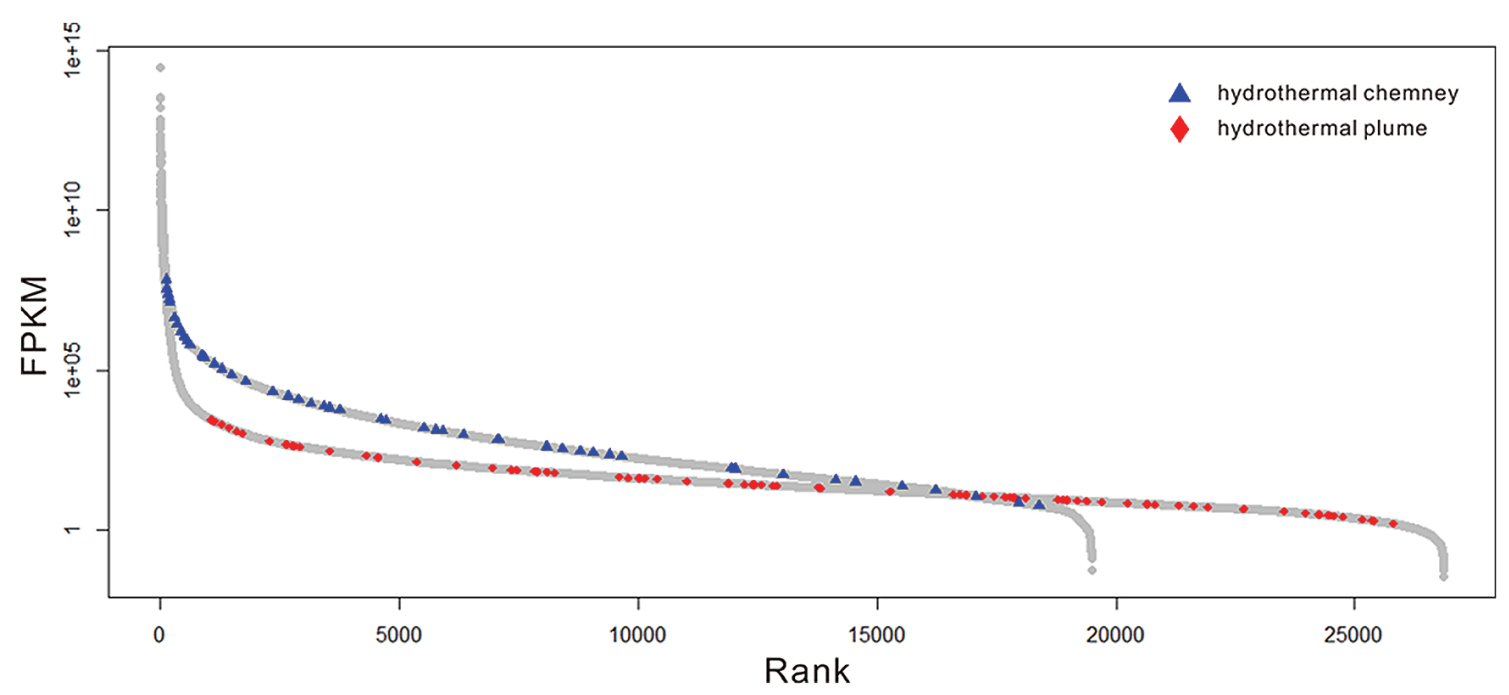

FIGURE 4 | Expression profiles of the genes in the metatranscriptome. The gray circles indicate the genes of the whole community. The blue triangles and red diamonds represent those related to sulfur oxidation and reduction in this metatranscriptome and a previous study (Lesniewski et al., 2012), respectively. The relative abundance of the gene transcripts was normalized to the length of the gene fragment and the total number of all of the transcripts.

may suggest that $\mathrm{H}_{2}$ or formate and play important roles in the flow of electrons during sulfate reduction. As shown above, the sulfur cycle in this community was particularly intensive and closely interacted with the carbon cycle, including carbon fixation and hydrocarbon degradation, to sustain the primary production in this ecosystem. 
TABLE 3 | Genes identified in the rTCA pathway in Delta- and Epsilonproteobacteria species.

\begin{tabular}{|c|c|c|c|c|}
\hline \multirow[t]{2}{*}{ Gene name } & \multirow[t]{2}{*}{ Abbrevations } & \multicolumn{2}{|c|}{ Assigned taxonomy* } & \multirow[t]{2}{*}{ FPKM $^{\#}$} \\
\hline & & Bin & BLAST & \\
\hline Malate dehydrogenase & $m d h$ & - & Bacteria & 424.81 \\
\hline Fumarate hydratase subunit alpha & fumA & $\operatorname{bin} 21$ & Desulfovibrionales & 355.77 \\
\hline Fumarate hydratase subunit beta & fumB & - & Bacteria & 135.08 \\
\hline Fumarate hydratase, class II & fumC & - & Bacteria & 0.00 \\
\hline Fumarate reductase, flavoprotein subunit & $\operatorname{frd} A$ & - & Desulfovibrionales & 1242.93 \\
\hline Fumarate reductase, iron-sulfur subunit & frdB & - & Epsilonproteobacteria & 7.09 \\
\hline Succinyl-CoA synthetase & sucC & & Bacteria & 57.34 \\
\hline Succinyl-CoA synthetase alpha subunit & sucD & - & Desulfobacterales & 724.00 \\
\hline 2-Oxoglutarate ferredoxin oxidoreductase subunit alpha & korA & - & Deltaproteobacteria & 65.56 \\
\hline 2-Oxoglutarate ferredoxin oxidoreductase subunit beta & korB & - & Epsilonproteobacteria & 232.91 \\
\hline 2-Oxoglutarate ferredoxin oxidoreductase subunit delta & korD & - & - & - \\
\hline 2-Oxoglutarate ferredoxin oxidoreductase subunit gamma & korC & - & Bacteria & 71.01 \\
\hline Isocitrate dehydrogenase & $i c d A$ & - & Bacteria & 93.51 \\
\hline Isocitrate dehydrogenase (NAD+) & $\mathrm{IDH} 3$ & - & Bacteria & 0.00 \\
\hline 2-Methylisocitrate dehydratase & $a c n B$ & - & Proteobacteria & 20.34 \\
\hline Aconitate hydratase & $\operatorname{acn} A$ & bin21 & Desulfovibrionales & 69.50 \\
\hline Aconitate hydratase 2 & $a c n B$ & - & Proteobacteria & 20.34 \\
\hline ATP-citrate lyase alpha-subunit & aclA & - & Epsilonproteobacteria & 238.10 \\
\hline ATP-citrate lyase beta-subunit & $a c / B$ & - & - & - \\
\hline Pyruvate ferredoxin oxidoreductase alpha subunit & porA & - & Epsilonproteobacteria & 9.90 \\
\hline Pyruvate ferredoxin oxidoreductase beta subunit & porB & - & Bacteria & 59.23 \\
\hline Pyruvate ferredoxin oxidoreductase delta subunit & porD & - & Bacteria & 2.66 \\
\hline Pyruvate ferredoxin oxidoreductase gamma subunit & porG & - & Epsilonproteobacteria & 44.25 \\
\hline ADP-forming acetyl-CoA synthetase & acd & bin21 & - & 136.79 \\
\hline Acetate kinase & ack & - & Thermotogaceae & 22.44 \\
\hline \multirow[t]{2}{*}{ Phosphate acetyltransferase } & pta & - & Caldisericaceae & 21.14 \\
\hline & & & & 424.81 \\
\hline
\end{tabular}

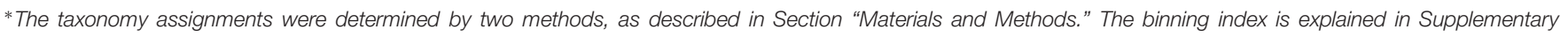
Table S1. \#FPKM is based on the maximal expression value of the annotated genes.

\section{Nitrogen Metabolism}

The key genes involved in the nitrogen metabolism were found, and some of these were found to be actively expressed (Table 4). Many Bacteria and Archaea have the potential to perform denitrification (Philippot, 2002), and numerous organic and inorganic compounds can be used as electron donors for denitrification. The genes involved in denitrification, including nar (nitrate reductase), nap (nitrate reductase), nir (nitrite reductase), nor (nitric oxide reductase) and nosZ, were found to be present in the metagenome. The nar $G$ gene was assigned to Beggiatoa, a nitrate-respiring and sulfide-oxidizing bacterium that has been found to dominate microbial mats in hydrothermal sediments in the Guaymas Basin (Winkel et al., 2014). narJ was found to be expressed in Alteromonadales, whereas napA and nap $B$ were found to be expressed in Epsilonproteobacteria. To summarize, a complete set of denitrification genes were found in the bacterial community of the chimney, though some of them were found at low expression levels (Table 4). Based on this observation, we propose that nitrogen denitrification present in this community is most likely mediated by Gammaproteobacteria and Epsilonproteobacteria, with electrons generated by the ORS pathway.

\section{DISCUSSION}

Since the discovery of the deep-sea hydrothermal ecosystem in 1977, it has been proposed that hydrogen sulfide-oxidizing chemoautotrophs may potentially sustain the primary production in these ecosystems (Kvenvolden et al., 1995), where hydrogen sulfide or sulfide is primarily supplied via the high temperatures of seawater-rock interactions in the subseafloor hydrothermal reaction zones (Jannasch and Mottl, 1985). The chemical and microbial oxidation and reduction reactions of sulfur compounds probably establish the overall sulfur metabolism in the ecosystem (Yamamoto and Takai, 2011). There is no doubt that the sulfur cycle is one of the most important microbial chemosynthetic pathways in the microbial habitats of hydrothermal vents, but few studies have attempted to characterize the process, particularly at the function and activity levels. To date, the mechanism through which a microbial community in hydrothermal fields can be fueled by sulfate metabolism remains unclear. In particular, metagenomic approaches have not been widely applied in studies of energy generation by the microbial sulfur cycle in hydrothermal systems. In this study, a combined metagenomic 
TABLE 4 | Genes identified in the nitrogen metabolic pathway in the microbial community.

\begin{tabular}{|c|c|c|c|c|}
\hline \multirow[t]{2}{*}{ Gene name } & \multirow[t]{2}{*}{ Abbrevations } & \multicolumn{2}{|c|}{ Assigned taxonomy* } & \multirow[t]{2}{*}{ FPKM $^{\#}$} \\
\hline & & Bin & BLAST & \\
\hline $\begin{array}{l}\text { Nitrate } \\
\text { reductase } \\
\text { alpha subunit }\end{array}$ & narG & - & Thiotrichales & 49.68 \\
\hline $\begin{array}{l}\text { Nitrate } \\
\text { reductase beta } \\
\text { subunit }\end{array}$ & narH & - & - & 2360.80 \\
\hline $\begin{array}{l}\text { Nitrate } \\
\text { reductase } \\
\text { gamma subunit }\end{array}$ & narl & - & Bacteria & 380.02 \\
\hline $\begin{array}{l}\text { Nitrate } \\
\text { reductase delta } \\
\text { subunit }\end{array}$ & narJ & - & Alteromonadales & 7.17 \\
\hline $\begin{array}{l}\text { Periplasmic } \\
\text { nitrate } \\
\text { reductase } \\
\text { NapA }\end{array}$ & napA & - & Epsilonproteobacteria & 98.48 \\
\hline $\begin{array}{l}\text { Cytochrome } \\
\text { c-type protein } \\
\text { NapB }\end{array}$ & nарв & - & Epsilonproteobacteria & 4.68 \\
\hline $\begin{array}{l}\text { Nitrite } \\
\text { reductase } \\
\text { (NO-forming) }\end{array}$ & nirk & - & - & - \\
\hline $\begin{array}{l}\text { Nitrite } \\
\text { reductase } \\
\text { (NO-forming) }\end{array}$ & nirs & - & - & - \\
\hline $\begin{array}{l}\text { Nitric oxide } \\
\text { reductase } \\
\text { subunit B }\end{array}$ & norB & - & Epsilonproteobacteria & 0.00 \\
\hline $\begin{array}{l}\text { Nitric oxide } \\
\text { reductase } \\
\text { subunit C }\end{array}$ & norC & - & - & -- \\
\hline $\begin{array}{l}\text { Nitrous-oxide } \\
\text { reductase }\end{array}$ & nosZ & - & Proteobacteria & 284.50 \\
\hline
\end{tabular}

* The taxonomy assignments were determined by two methods, as described in Section "Materials and Methods." The binning index is explained in Supplementary Table S1. \#FPKM is based on the maximal expression value of the annotated genes.

and metatranscriptomic study of a chimney in the Guaymas Basin provides insight into the complete sulfur cycle based on the results from not only the genomic but also the expression analysis, the combination of which has not been previously used for the analysis of a deep-sea hydrothermal vent chimney sample.

The accumulation of hydrogen sulfides at the outer chimney promoted the coupling of sulfide oxidation to the electron acceptors present in the nearby marine water, including oxygen and nitrate, as supported by the retrieval of the functional and expressed genes described herein (Tables 2-4 and Figure 3). These findings suggest that the coupling between sulfur oxidation and denitrification may fuel some $\mathrm{N}$-metabolizing microorganisms at the sulfide-enriched outer chimney. As proposed in this study, the microorganisms involved in this process were Epsilonproteobacteria as the sulfur-oxidizing bacteria, and Gammaproteobacteria and Epsilonproteobacteria as potential denitrifiers. The other sulfur-metabolizing group, namely sulfate-reducing prokaryotes, may use hydrogen and/or dissolved organic matter as electron donors, as hydrogenases and key genes for the degradation of organic compounds have been identified in this study (Supplementary Tables S5 and S6).

Carbon fixation pathways other than the Calvin-BensonBassham (CBB) cycle have been found to exhibit a notable contribution to carbon fixation, mostly at deep-sea hydrothermal vents (Campbell and Cary, 2004). The rTCA cycle was found to be highly expressed in the dominant Delta- and Epsilonproteobacteria. The key enzyme for the utilization of acetate was also identified to be expressed in this study (Table 3). Generally, the rTCA cycle appears to be dominant in habitats with a temperature ranging from 20 to $90^{\circ} \mathrm{C}$, whereas the CBB cycle and the Wood-Ljungdahl (WL) pathway may be the principal pathways at temperatures lower than $20^{\circ} \mathrm{C}$ and greater than $90^{\circ} \mathrm{C}$, respectively (Hugler and Sievert, 2011). In the present sample, the $\mathrm{CBB}$ cycle was not found present, which is consistent with the fact that this sample was collected from a high-temperature condition ( $\mathrm{He}$ et al., 2013). In addition, the enzymes for the degradation of a variety of organic compounds, such as hydrocarbons, fatty acids, chitins and proteins, have been detected at both DNA and RNA level (Supplementary Table S5). Together, all of these organic compounds may be the carbon source for this microbial community.

In this scenario, both autotrophic and heterotrophic SRB could inhabit the inner chimney (Figure 3), where sulfate reduction is coupled to carbon fixation and hydrocarbon oxidation. Based on the expression levels of key genes in rTCA (Table 3) and alkane degradation (Supplementary Table S4), hydrocarbon degradation might contribute substantially to the linking of $\mathrm{S}$ and $\mathrm{C}$ cycle at inner layer chimney. In another word, heterotrophic SRB, commonly found at vent systems, may be the major player in coordinating and influencing the $S$ and $\mathrm{C}$ cycle. Compared the expression of key genes in sulfur metabolizing and the rest processes (Figure 4), the reduced sulfur would be quickly and intensively oxidized to fuel the community, where sulfate-reducing microbes were found dominated. The composition of the sulfate-reducing community was determined by the way that microbes perform carbon metabolism. In our sample, heterotrophic SRB was found prevalent with their capabilities in hydrocarbon degradation. This finding may improve our understanding on the structure, function, and interaction within microbial community in hydrothermal vent.

Meta-omics based approaches have the advantages in studying the entire microbial community without pure cultures or prior knowledge on the sample. Functional omics approaches, such as transcriptome and proteome, could further confirm the metabolic potential at the active level. More efforts will be spent on quantification and comparison of these function omics datasets. Together with in situ carbon stable isotope measurement, and lipid type and diversity analysis, the activity, rate and interaction of key process in a given environmental condition could be accessed and estimated. 


\section{MATERIALS AND METHODS}

\section{Sample Collection and Processing}

The sample 4558-6 under investigation was collected from the outer layer of a black-smoker chimney in the Guaymas Basin and was previously described through a metagenome-based study (He et al., 2013). The sample was fixed with RNAlater (SigmaAldrich, Munich, Germany) and stored at $-80^{\circ} \mathrm{C}$ prior to DNA and RNA extraction. DNA isolation was conducted as described previously (Wang et al., 2013). Metagenome pyrosequencing was performed using a 454 Life Sciences GS FLX system with a practical limit of $400 \mathrm{bp}$. RNA was isolated with a RNA isolation kit (Omega Bio-Tek, Doraville, GA, USA) following the user's manual provided by the manufacturer. RNA samples were treated with DNAse (Thermo) for $45 \mathrm{~min}$ at $37^{\circ} \mathrm{C}$, and then used as a template for PCR to detect undigested DNA. The mRNA fraction was enriched through the enzymatic digestion of rRNA molecules (mRNA-ONLY Prokaryotic mRNA Isolation kit, Epicentre Biotechnologies, Madison, WI, USA) followed by the subtractive hybridization of rRNA with capture oligonucleotides (Ambion MICROBExpress kit, Life Technologies, Gaithersburg, MD, USA). The mRNA isolates were first amplified (MessageAmp II-Bacteria kit, Ambion, Life Technologies) and then reversely transcribed into complementary DNA. Afterward, the cDNA was directly sequenced using the Illumina (BGI-Shenzhen, China) Hiseq2000 platform (2*90 bp pair-end) for metatranscriptome analysis.

\section{Metagenome Assembly and Annotation}

The reads obtained through metagenome sequencing were assembled and annotated as previously described (He et al., 2013). Briefly, low quality sequencing reads were trimmed in Geneious 6.04 (Biomatters Ltd.) and technical replicates were removed with cd-hit (at 96\% sequence identity; Fu et al., 2012). After removing short reads $(<100 \mathrm{bp})$, the remaining reads were assembled with Velvet (Zerbino and Birney, 2008). Coding regions of the metagenomic assembly were predicted using FragGeneScan (Rho et al., 2010) and then BLASTed (Altschul et al., 1997; $1 \mathrm{e}^{-5}$ ) against an NCBI non-redundant (NR) protein database. The $16 \mathrm{~S}$ rRNA genes were picked using Sortmerna and BLASTed against GreenGene database (e-value $<1 \mathrm{e}^{-5}$ ) respectively. For functional annotation, sequences with matches to the COG (Tatusov et al., 2003), Pfam (Finn et al., 2014), and KEGG (Ogata et al., 1999) databases were retrieved to establish the functional categories and reconstruct the metabolic pathways. The genes of interest, such as transposases, were subjected to manual checkup, and spurious annotations (putative, like-, similar to) were excluded from further analysis.

\section{Taxonomic Assignment}

Two different methods were applied to assess the taxonomic information. First, the assembled metagenomic sequences was binned using the tetranucleotide frequencies in emergent self-organizing maps (ESOMs; Dick et al., 2009) with a window size of $8 \mathrm{kbp}$, a sliding window size of $4 \mathrm{kbp}$, and the minimum fragment size of $2 \mathrm{kbp}$. Complete genomic sequences of 20 species were used as references (designated as bin1-20), these microorganism were listed as following: Acinetobacter pittii ANC 4052, Alteromonas macleodii str. 'Deep ecotype', Candidatus Pelagibacter ubique HTCC1062, uncultured marine crenarchaeote E37-7F, Marine group II euryarchaeote SCGC AAA288-C18, Marine Group II euryarchaeote SCGC AB-629-J06, uncultured marine group II euryarchaeote (marine metagenome), Marine Group III euryarchaeote SCGC AAA007-O11, Marine Group III euryarchaeote SCGC AAA288-E19, Marinobacter nanhaiticus D15-8W, Methylobacter tundripaludum SV96, Methylophaga aminisulfidivorans MP, Methylotenera mobilis JLW8, Nitrosopumilus maritimus SCM1, Candidatus Nitrospira defluvii, Planctopirus limnophila DSM 3776, Pseudomonas denitrificans ATCC 13867, Candidatus Ruthia magnifica str. Cm (Calyptogena magnifica), SAR324 cluster bacterium SCGC AAA240-J09 and SAR86 cluster bacterium SAR86E. After binning, the completeness and taxonomic classification of the genomes within bins were then estimated by counting and BLASTing universal single-copy genes as previously described (Rinke et al., 2013). Alternatively, each predicted sequence feature in the metagenome and metatranscriptome was assigned to a certain taxon if at least 75\% of the BLAST hits of this query were from that specific taxon. A BLAST search of all of the reads against the non-redundant protein database in NR was performed. All of the hits obtained from the BLAST searches were retained, and their taxonomic affiliations were determined using MEGAN (Huson et al., 2007) with bit-score values of 100 . The taxonomic compositions of each predicted gene feature was then visualized using MEGAN.

\section{Metatranscriptome Mapping and Transcript Quantification}

The raw shotgun sequencing metatranscriptomic reads obtained by Illumina pair-end sequencing were dereplicated (100\% identity over $100 \%$ lengths) and trimmed using sickle ${ }^{1}$. The dereplicated, trimmed, and paired-end Illumina reads were then mapped to the metagenome using Bowtie (Langmead and Salzberg, 2012) with the default parameters. The unique mapped reads were selected, and FPKM (expected fragments per kilobase of transcript per million fragments mapped) was used to estimate the expression level of each gene using a script downloaded from $\mathrm{GitHub}^{2}$.

\section{Estimation of the Completeness of Genomic Bins}

The complete genome sizes of the genomic bins were estimated based on an analysis of conserved single-copy genes (CSCGs) as described by Lloyd et al. (2013). In total, we were able to collect 162 and 139 universal CSCGs for the archaea and bacteria genomes, as in the previous study (Rinke et al., 2013). The ratios between the numbers of CSCGs present in the metagenome and the number of total CSCGs were then used to estimate the size of each genome bin.

\footnotetext{
${ }^{1}$ https://github.com/najoshi/sickle

${ }^{2}$ https://github.com/minillinim/sam2FPKG
} 


\section{Comparative Analysis}

The expression patterns of the sulfur-metabolizing genes in this metatranscriptome were compared to those in the metatranscriptome of a plum sample from Guaymas Basin (Lesniewski et al., 2012). Comparisons between two metatranscriptomes were conducted using the Mann-Whitney $U$-test. The gene expression profiles were compared between two samples using the normalized rank from 0 to 1 in each respective sample as the input. A difference was considered significant if the $p$-value was lower than 0.001 .

\section{Construction of a Phylogenetic Tree}

The predicted sequence features were checked across multiple annotation databases and then aligned with ClustalW (Larkin et al., 2007), and any gaps were removed manually. To construct functional gene phylogenies, the aligned sequences were analyzed by maximum likelihood-based FastTree (Price et al., 2010) using the Jones-Taylor-Thornton (JTT) with CAT approximation.

\section{Metabolic Pathway Identification}

The gene products were searched for similarity against the KEGG database. A match was counted if the similarity search resulted in an expectation e-value below $1 \mathrm{e}^{-5}$. All of the occurring $\mathrm{KO}$ (KEGG Orthology) numbers were mapped against the KEGG pathway functional hierarchies and the COG database. For genes

\section{REFERENCES}

Agrawal, A., and Gieg, L. M. (2013). In situ detection of anaerobic alkane metabolites in subsurface environments. Front. Microbiol. 4:140. doi: 10.3389/fmicb. 2013.00140

Altschul, S. F., Madden, T. L., Schaffer, A. A., Zhang, J., Zhang, Z., Miller, W., et al. (1997). Gapped BLAST and PSI-BLAST: a new generation of protein database search programs. Nucleic Acids Res. 25, 3389-3402. doi: 10.1093/nar/25. 17.3389

Anantharaman, K., Breier, J. A., Sheik, C. S., and Dick, G. J. (2013). Evidence for hydrogen oxidation and metabolic plasticity in widespread deep-sea sulfur-oxidizing bacteria. Proc. Natl. Acad. Sci. U.S.A. 110, 330-335. doi: $10.1073 /$ pnas. 1215340110

Baumberger, T., Lilley, M. D., Resing, J. A., Lupton, J. E., Baker, E. T., Butterfield, D. A., et al. (2014). Understanding a submarine eruption through time series hydrothermal plume sampling of dissolved and particulate constituents: West Mata, 2008-2012. Geochem. Geophys. Geosyst. 15, 4631-4650. doi: 10.1002/2014GC005460

Bergmann, F. D., Selesi, D., and Meckenstock, R. U. (2011). Identification of new enzymes potentially involved in anaerobic naphthalene degradation by the sulfate-reducing enrichment culture N47. Arch. Microbiol. 193, 241-250. doi: 10.1007/s00203-010-0667-4

Biddle, J. F., Cardman, Z., Mendlovitz, H., Albert, D. B., Lloyd, K. G., Boetius, A., et al. (2012). Anaerobic oxidation of methane at different temperature regimes in Guaymas Basin hydrothermal sediments. ISME J. 6, 1018-1031. doi: 10.1038/ismej.2011.164

Brazelton, W. J., and Baross, J. A. (2010). Metagenomic comparison of two Thiomicrospira lineages inhabiting contrasting deep-sea hydrothermal environments. PLoS ONE 5:e13530. doi: 10.1371/journal.pone.0013530

Campbell, B. J., and Cary, S. C. (2004). Abundance of reverse tricarboxylic acid cycle genes in free-living microorganisms at deep-sea hydrothermal vents. Appl. Environ. Microbiol. 70, 6282-6289. doi: 10.1128/AEM.70.10.6282-6289.2004

Cao, H., Wang, Y., Lee, O. O., Zeng, X., Shao, Z., and Qian, P. Y. (2014). Microbial sulfur cycle in two hydrothermal chimneys on the Southwest Indian Ridge. MBio 5, e980-13. doi: 10.1128/mBio.00980-13 with multiple hits, only the genes with the highest expression value (FPKM) are displayed in the figures and tables and further discussed in the text.

\section{Data Availability}

The metatranscriptome sequences are available on NCBI as SRX1008212. The assembled sequence was uploaded to IMG with a project ID Ga0072503.

\section{ACKNOWLEDGMENTS}

We thank Anna-Louise Reysenbach for providing the chance to attend the expedition, and all the crew members from AT-26 cruise. This work was supported by National High Technology Research and Development Program of China (Grant No. 2012AA092103), China Ocean Mineral Resources $\mathrm{R} \& \mathrm{D}$ Association (Grant No.DY125-22-04 and DY12515-T-04).

\section{SUPPLEMENTARY MATERIAL}

The Supplementary Material for this article can be found online at: http://journal.frontiersin.org/article/10.3389/fmicb. 2015.01236

Dick, G. J., Andersson, A. F., Baker, B. J., Simmons, S. L., Thomas, B. C., Yelton, A. P., et al. (2009). Community-wide analysis of microbial genome sequence signatures. Genome Biol. 10, R85. doi: 10.1186/gb-2009-10-8-r85

Finn, R. D., Bateman, A., Clements, J., Coggill, P., Eberhardt, R. Y., Eddy, S. R., et al. (2014). Pfam: the protein families database. Nucleic Acids Res. 42, D222-D230. doi: $10.1093 /$ nar/gkt1223

Fornari, D. J., Shank, T. M., Von Damm, K. L., Gregg, T. K. P., Lilley, M. D., Levai, G., et al. (1998). Time-series temperature measurements at hightemperature hydrothermal vents, East Pacific Rise $9^{\circ} 49^{\prime}-51^{\prime} \mathrm{N}$ : monitoring a crustal cracking event. Earth Planet. Sci. Lett. 160, 419-430. doi: 10.1016/S0012821X(98)00101-0

Fritz, G., Roth, A., Schiffer, A., Buchert, T., Bourenkov, G., Bartunik, H. D., et al. (2002). Structure of adenylylsulfate reductase from the hyperthermophilic Archaeoglobus fulgidus at 1.6-A resolution. Proc. Natl. Acad. Sci. U.S.A. 99, 1836-1841. doi: 10.1073/pnas.042664399

Fu, L., Niu, B., Zhu, Z., Wu, S., and Li, W. (2012). CD-HIT: accelerated for clustering the next-generation sequencing data. Bioinformatics 28, 3150-3152. doi: 10.1093/bioinformatics/bts565

He, Y., Xiao, X., and Wang, F. (2013). Metagenome reveals potential microbial degradation of hydrocarbon coupled with sulfate reduction in an oilimmersed chimney from Guaymas Basin. Front. Microbiol. 4:148. doi: $10.3389 /$ fmicb.2013.00148

Hugler, M., and Sievert, S. M. (2011). Beyond the Calvin cycle: autotrophic carbon fixation in the ocean. Ann. Rev. Mar. Sci. 3, 261-289. doi: 10.1146/annurevmarine-120709-142712

Huson, D. H., Auch, A. F., Qi, J., and Schuster, S. C. (2007). MEGAN analysis of metagenomic data. Genome Res. 17, 377-386. doi: 10.1101/gr. 5969107

Jannasch, H. W., and Mottl, M. J. (1985). Geomicrobiology of deepsea hydrothermal vents. Science 229, 717-725. doi: 10.1126/science.229. 4715.717

Kvenvolden, K. A., Hostettler, F. D., Carlson, P. R., Rapp, J. B., Threlkeld, C. N., and Warden, A. (1995). Ubiquitous tar balls with a california-source signature on the shorelines of prince william sound, alaska. Environ. Sci. Technol. 29, 2684-2694. doi: 10.1021/es00010a033 
Langmead, B., and Salzberg, S. L. (2012). Fast gapped-read alignment with Bowtie 2. Nat. Methods 9, 357-359. doi: 10.1038/nmeth.1923

Larkin, M. A., Blackshields, G., Brown, N. P., Chenna, R., Mcgettigan, P. A., Mcwilliam, H., et al. (2007). Clustal W and Clustal X version 2.0. Bioinformatics 23, 2947-2948. doi: 10.1093/bioinformatics/btm404

Lesniewski, R. A., Jain, S., Anantharaman, K., Schloss, P. D., and Dick, G. J. (2012). The metatranscriptome of a deep-sea hydrothermal plume is dominated by water column methanotrophs and lithotrophs. ISME J. 6, 2257-2268. doi: 10.1038/ismej.2012.63

Lloyd, K. G., Schreiber, L., Petersen, D. G., Kjeldsen, K. U., Lever, M. A., Steen, A. D., et al. (2013). Predominant archaea in marine sediments degrade detrital proteins. Nature 496, 215-218. doi: 10.1038/nature12033

Mino, S., Kudo, H., Arai, T., Sawabe, T., Takai, K., and Nakagawa, S. (2014). Sulfurovum aggregans sp. nov., a hydrogen-oxidizing, thiosulfatereducing chemolithoautotroph within the Epsilonproteobacteria isolated from a deep-sea hydrothermal vent chimney, and an emended description of the genus Sulfurovum. Int. J. Syst. Evol. Microbiol. 64, 3195-3201. doi: 10.1099/ijs.0.065094-0

Nakagawa, S., Takai, K., Inagaki, F., Hirayama, H., Nunoura, T., Horikoshi, K., et al. (2005). Distribution, phylogenetic diversity and physiological characteristics of epsilon-Proteobacteria in a deep-sea hydrothermal field. Environ. Microbiol. 7, 1619-1632. doi: 10.1111/j.1462-2920.2005.00856.x

Ogata, H., Goto, S., Sato, K., Fujibuchi, W., Bono, H., and Kanehisa, M. (1999). KEGG: kyoto encyclopedia of genes and genomes. Nucleic Acids Res. 27, 29-34. doi: 10.1093/nar/27.1.29

Orcutt, B. N., Sylvan, J. B., Knab, N. J., and Edwards, K. J. (2011). Microbial ecology of the dark ocean above, at, and below the seafloor. Microbiol. Mol. Biol. Rev. 75, 361-422. doi: 10.1128/MMBR.00039-10

Philippot, L. (2002). Denitrifying genes in bacterial and Archaeal genomes. Biochim. Biophys. Acta 1577, 355-376. doi: 10.1016/S0167-4781(02) 00420-7

Price, M. N., Dehal, P. S., and Arkin, A. P. (2010). FastTree 2-approximately maximum-likelihood trees for large alignments. PLOS ONE 5:e9490. doi: 10.1371/journal.pone.0009490

Reysenbach, A. L., and Shock, E. (2002). Merging genomes with geochemistry in hydrothermal ecosystems. Science 296, 1077-1082. doi: 10.1126/science. 1072483

Rho, M., Tang, H., and Ye, Y. (2010). FragGeneScan: predicting genes in short and error-prone reads. Nucleic Acids Res. 38, e191. doi: 10.1093/nar/gkq747

Rinke, C., Schwientek, P., Sczyrba, A., Ivanova, N. N., Anderson, I. J., Cheng, J. F., et al. (2013). Insights into the phylogeny and coding potential of microbial dark matter. Nature 499, 431-437. doi: 10.1038/nature12352
Rueter, P., Rabus, R., Wilkes, H., Aeckersberg, F., Rainey, F. A., Jannasch, H. W., et al. (1994). Anaerobic oxidation of hydrocarbons in crude oil by new types of sulphate-reducing bacteria. Nature 372, 455-458. doi: 10.1038/ $372455 \mathrm{a} 0$

Sylvan, J. B., Sia, T. Y., Haddad, A. G., Briscoe, L. J., Toner, B. M., Girguis, P. R., et al. (2013). Low temperature geomicrobiology follows host rock composition along a geochemical gradient in lau basin. Front. Microbiol. 4:61. doi: 10.3389/fmicb.2013.00061

Tatusov, R. L., Fedorova, N. D., Jackson, J. D., Jacobs, A. R., Kiryutin, B., Koonin, E. V., et al. (2003). The COG database: an updated version includes eukaryotes. BMC Bioinformatics 4:41. doi: 10.1186/1471-2105-4-41

Teske, A., Callaghan, A. V., and Larowe, D. E. (2014). Biosphere frontiers of subsurface life in the sedimented hydrothermal system of Guaymas Basin. Front. Microbiol. 5:362. doi: 10.3389/fmicb.2014.00362

Von Damm, K. L. (1990). Seafloor hydrothermal activity: black smoker chemistry and chimneys. Annu. Rev. Earth Planet. Sci. 18, 173-204. doi: 10.1111/gbi. 12086

Wang, J., Shen, J., Wu, Y., Tu, C., Soininen, J., Stegen, J. C., et al. (2013). Phylogenetic beta diversity in bacterial assemblages across ecosystems: deterministic versus stochastic processes. ISME J. 7, 1310-1321. doi: 10.1038/ismej.2013.30

Winkel, M., De Beer, D., Lavik, G., Peplies, J., and Mussmann, M. (2014). Close association of active nitrifiers with Beggiatoa mats covering deep-sea hydrothermal sediments. Environ. Microbiol. 16, 1612-1626. doi: 10.1111/14622920.12316

Yamamoto, M., and Takai, K. (2011). Sulfur metabolisms in epsilon- and gammaProteobacteria in deep-sea hydrothermal fields. Front. Microbiol. 2:192. doi: 10.3389/fmicb.2011.00192

Zerbino, D. R., and Birney, E. (2008). Velvet: algorithms for de novo short read assembly using de Bruijn graphs. Genome Res. 18, 821-829. doi: 10.1101/gr.074492.107

Conflict of Interest Statement: The authors declare that the research was conducted in the absence of any commercial or financial relationships that could be construed as a potential conflict of interest.

Copyright (c) 2015 He, Feng, Fang, Zhang and Xiao. This is an open-access article distributed under the terms of the Creative Commons Attribution License (CC BY). The use, distribution or reproduction in other forums is permitted, provided the original author(s) or licensor are credited and that the original publication in this journal is cited, in accordance with accepted academic practice. No use, distribution or reproduction is permitted which does not comply with these terms. 